\title{
Recent Results from Beam Tests of 3D and Pad pCVD Diamond Detectors
}

\author{
Rainer Wallny* for the RD42 Collaboration ${ }^{\dagger}$ \\ ETH Zurich \\ E-mail: rainer.wallnyaphys .ethz .ch
}

\begin{abstract}
Results from prototypes of a detector using chemical vapor deposited (CVD) diamond with embedded resistive electrodes in the bulk forming a 3D diamond device are presented. A detector system consisting of 3D devices based on poly-crystalline CVD (pCVD) diamond was connected to a multi-channel readout and successfully tested in a $120 \mathrm{GeV} / \mathrm{c}$ proton beam at CERN proving for the first time the feasibility of the 3D detector concept in pCVD for particle tracking applications. We also present beam test results on the dependence of signal size on incident particle rate in charged particle detectors based on poly-crystalline CVD diamond. The detectors were tested in a $260 \mathrm{MeV} / \mathrm{c}$ pion beam over a range of particle fluxes from $2 \mathrm{kHz} / \mathrm{cm}^{2}$ to $10 \mathrm{MHz} / \mathrm{cm}^{2}$. The pulse height of the sensors was measured with pad readout electronics at a peaking time of $7 \mathrm{~ns}$. Our data from the 2015 beam tests at PSI indicate that the pulse height of poly-crystalline CVD diamond sensor irradiated to $5 \times 10^{14} \mathrm{n}_{\mathrm{eq}} / \mathrm{cm}^{2}$ is independent of particle flux at $3 \%$ level.
\end{abstract}

38th International Conference on High Energy Physics

3-10 August 2016

Chicago, USA

\footnotetext{
*Speaker.

${ }^{\dagger}$ The full list of RD42 authors is provided in the Appendix.
} 


\section{Introduction}

With the planned upgrade of the Large Hadron Collider (LHC) to the High Luminosity LHC (HLLHC) by 2026 [1], the inner tracking detectors will be exposed to a total fluence of $2 \times 10^{16} \mathrm{n}_{\text {eq }} / \mathrm{cm}^{2}$ over their lifetime. The LHC experiments are therefore tasked to develop tracking technology that will ensure reliable operation of the detectors up to this fluence. Diamond is considered as one of the candidate technologies to provide sufficiently radiation tolerant sensors. Diamond also offers, amongst others, the distinct advantage of low leakage currents even after exposure to a high irradiation dose which allows operation without cooling and thus easing the requirements on the support infrastructure.

The RD42 collaboration at CERN is working to develop radiation tolerant devices based on poly-crystalline and single-crystal Chemical Vapor Deposition (pCVD and scCVD) diamond as sensor material. In addition to planar sensors, RD42 recently demonstrated that the 3D sensor concept can be successfully implemented in pCVD and scCVD diamonds [2]. In 3D diamond sensors, the readout and bias electrodes are embedded inside the sensor bulk perpendicular to the surface of the sensor. A 3D device fabricated out of scCVD diamond was able to collect full charge at much lower bias voltage than the planar device fabricated out of the same diamond [3]. Similarly, the 3D device made out of pCVD diamond, described in section 2 of this paper, was able to collect more charge at lower bias than the planar device made out of the same diamond. In addition to developing new device concepts using $\mathrm{pCVD}$ and scCVD diamond, RD42 is working to study the properties of diamond sensors further. A current priority is the study of the high rate behavior of pCVD and scCVD diamond sensors. New results of this study are presented in section 3.

\section{3D diamond detector beam tests at CERN}

The 3D device [4] was fabricated using a $5 \mathrm{~mm} \times 5 \mathrm{~mm} \times 0.5 \mathrm{~mm}$ pCVD diamond. Fig. 1a) shows a microscope photograph of the finished device and Fig. 1b) shows the design mask pattern used for metalization. The test device implemented multiple metalization patterns: a planar strip detector with a $50 \mu \mathrm{m}$ strip pitch, a 3D detector with a row of resistive electrodes connected by a metal strip to either readout or bias detector and a 3D phantom detector, where the same readout metalization pattern was used as in the case of the 3D detector but without the bulk electrodes. The electrodes were fabricated by exposing the diamond to a femtosecond laser radiation inducing a transition of the bulk material from the diamond phase to a phase that consisted of a mixture of diamond-likecarbon, amorphous carbon and graphitic material $[3,5]$. The connections to the electrodes of the 3D device were made with $\mathrm{Cr} / \mathrm{Au}$ metals using a photo-lithographic process. The details of the fabrication are similar to the device described in Ref. [3], where more information can be found.

The metalized sensor was wire-bonded to a VA2.2 readout chip [6]. The completed detector was then installed as detector under test (DUT) into a high resolution beam telescope at the H6A secondary beam line of Super-Proton-Synchrotron (SPS) complex at CERN. The beam line was tuned to provide $120 \mathrm{GeV} / \mathrm{c}$ protons with an average flux of $\sim 10 \mathrm{kHz} / \mathrm{cm}^{2}$. The pulse height of the beam particles in the detector was measured for a set of positive and negative bias voltages. In order to assess the performance of the detector, the noise of every connected channel was measured in events with no hit on that channel. Fig. 2b) shows that the noise performance of the planar and 
the 3D phantom detector is approximately the same. The noise in the 3D detector, after exclusion of four noisy strips is about $17 \%$ higher than the planar or 3D phantom detector which is likely due to a larger capacitance of the resistive columns of the 3D detector.

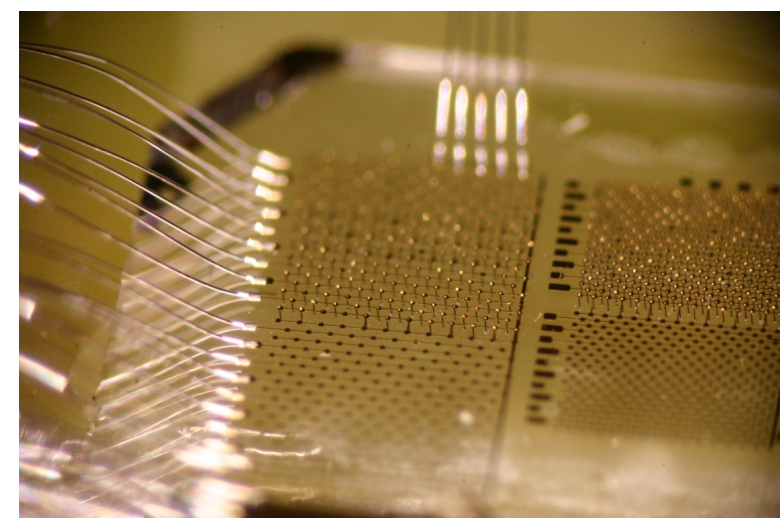

(a) Photo of metalization pattern

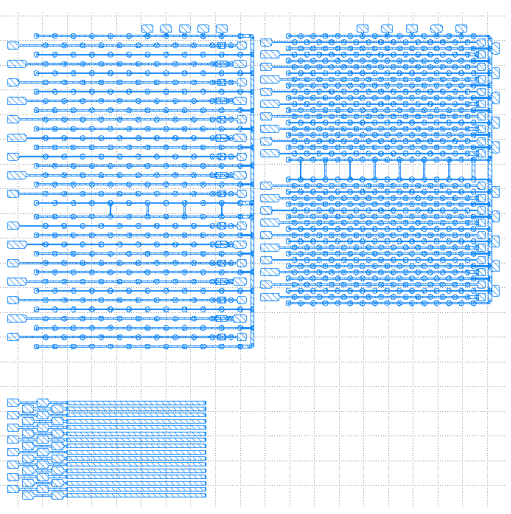

(b) Metalization mask

Figure 1: A picture of a) the 3D device and b) metalization mask. The test device consists of a planar strip detector with a $50 \mu \mathrm{m}$ strip pitch (b, bottom left), a 3D detector connected by a metal strip to either readout or bias detector (b, top left) and a 3D phantom detector (b, middle left), where the same metalization pattern was used as in the case of the 3D detector but without the bulk electrodes. The structures shown on the right of a) and b) were not tested in this beam test.

After reconstructing the hits in the telescope and the DUT the first $10 \%$ of the events were used to align the telescope and the DUT. The rest of the events were then used in the analysis. The track was projected into the plane of the DUT and the average pulse height in the DUT was plotted versus the predicted hit position. The result is shown in Fig. 2a). Qualitatively it can be observed that the 3D device provides more charge than the planar device. The dark square-like regions in the 3D detector pulse height map are caused by non-working bias or readout electrodes [2].

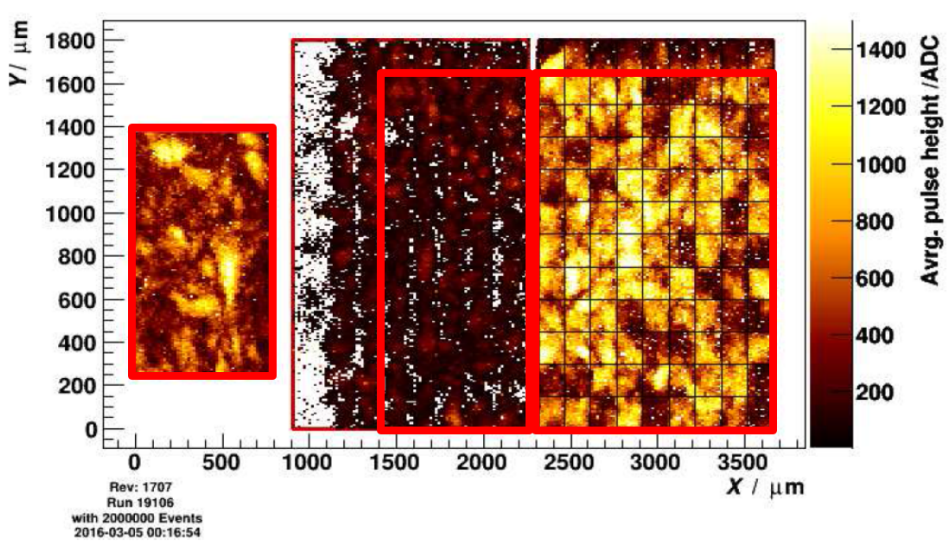

(a)

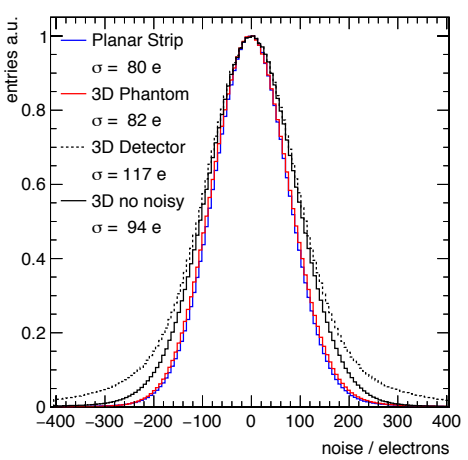

(b)

Figure 2: a) Average pulse height versus predicted hit position in the strip, 3D phantom and 3D pattern (from left to right). b) Noise in electron equivalent for the three device patterns and for the 3D detector without 4 noisy channels. 


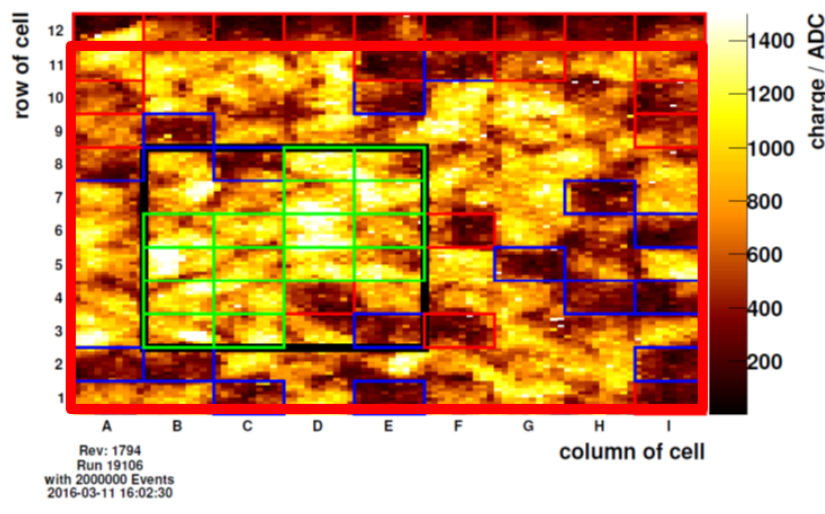

(a)

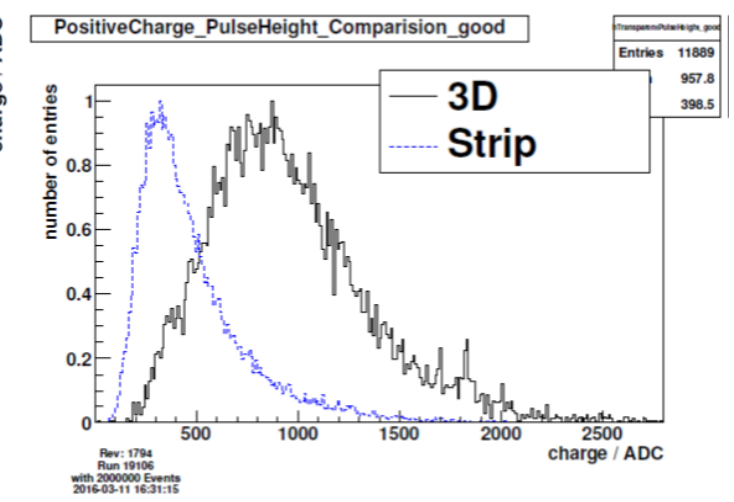

(b)

Figure 3: a) Contiguous fiducial region in the 3D detector used for the analysis (marked in green). Cells with a broken electrode are marked in blue (readout) and red (HV). b) Pulse height distributions of the 3D detector (a) compared with the pulse height distribution of the strip detector measured with the same electronics.

In order to disentangle the column production efficiency from the 3D detector performance in comparison to the planar strip device, a continuous fiducial region of working cells was selected (Fig. 3a). The pulse height spectrum from this fiducial region in the 3D detector and the pulse height spectrum of the strip detector is shown in Fig. 3b). The average charge collected by the $3 \mathrm{D}$ device is about twice as large as the strip detector. Recently further progress was made in fabricating 3D electrodes allowing us to build much larger structures (about 1000 3D cells). This pCVD 3D detector was recently measured in the same beam line as above and results are being analyzed. First preliminary results indicate (in selected regions of the 1000 cell device) a mean pulse height approaching (85\%) of the full charge collection, indicating the potential of the 3D pattern technique for diamond sensors.

\section{Pulse height vs rate study of pCVD pad detectors at PSI}

The study pulse height dependence in the CVD diamond sensors on the particle flux was prompted by the experience [7] in the pilot run of the Pixel Luminosity Telescope (PLT) [8]. For our study the $\pi \mathrm{M} 1$ beam line of the High Intensity Proton Accelerator (HIPA) at Paul Scherrer Institute (PSI) was chosen. It is able to deliver controllable $260 \mathrm{MeV} / \mathrm{c} \pi^{+}$fluxes from a few $\mathrm{kHz} / \mathrm{cm}^{2}$ to a few $\mathrm{MHz} / \mathrm{cm}^{2}$ in bunches spaced by $19.8 \mathrm{~ns}$ [9]. Since 2013, RD42 has performed several successful beam tests at this facility and we report here the latest results. The measurement setup consisted of a beam telescope for particle tracking and two DUTs. The beam telescope comprises four tracking planes, two planes positioned upstream of the DUTs and two downstream. Each plane consisted of pixelated silicon sensors with pixel pitch of $100 \mu \mathrm{m} \times 150 \mu \mathrm{m}$ read out by a PSI46v2 readout chip [10]. A coincidence of the two telescope planes closest to the DUTs and a scintillator located $\sim 10 \mathrm{~cm}$ downstream of the last telescope plane was used for triggering. The use of the pixel planes for triggering reduced the trigger area to approximately the fiducial area of the DUTs, while the scintillator determined the timing of particles with precision of $0.7 \mathrm{~ns}$. Each 
DUT plane was a $5 \mathrm{~mm} \times 5 \mathrm{~mm} \times 0.5 \mathrm{~mm}$ pCVD diamond sensor produced by II-VI Incorporated [11] with $\mathrm{Cr} / \mathrm{Au}$ electrodes patterned by a lift off process. The size of both the front and back electrodes of this pad detector was $3.5 \mathrm{~mm} \times 3.5 \mathrm{~mm}$ and the front electrode was surrounded by a guard ring. The back of the sensor was glued with a conductive epoxy to a PCB, while the front was wire-bonded to a fast spectroscopic amplifier [12] with $7 \mathrm{~ns}$ rise time and $23 \mathrm{~ns}$ fall time.

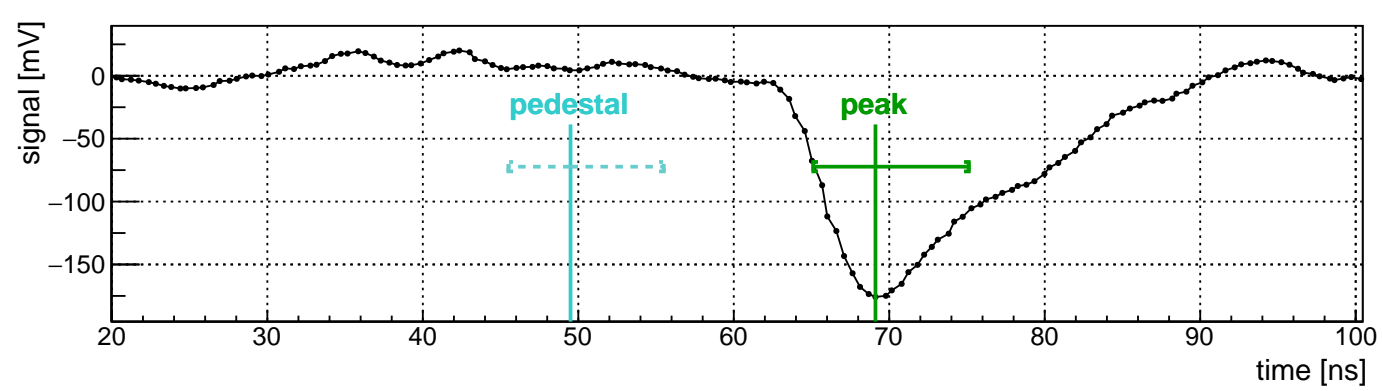

Figure 4: A sample signal trace. The horizontal bars indicate the averaging regions for pedestal (dashed) and for signal (solid).

The amplified signals were recorded with a DRS4 evaluation board [13] at 2 GS per second. Fig. 4 shows a portion of the DRS4 trace with a typical signal. The full trace is $\sim 500 \mathrm{~ns}$ long and can contain signals from particles that came from a bunch other than the one that generated the trigger. In order to determine the relative timing of the signal with respect to the trigger particle several waveforms were averaged and the most probable peak time was chosen as the trigger time. In order to extract the magnitude of the signal the waveforms were time averaged in a $10 \mathrm{~ns}$ window (indicated by the horizontal green line in Fig. 4 and, to extract the pedestal, 4 ns before the peak and $6 \mathrm{~ns}$ after the peak. The pedestal region (dashed aqua-colored line in Fig. 4) was set to exactly one bunch $(19.8 \mathrm{~ns})$ before the mean time of the signal as the probability of a signal contamination in this bunch was minimal due to the trigger logic. The $10 \mathrm{~ns}$ time windows optimize the signal to noise ratio.

After all analysis cuts are applied no pedestal events remain in the signal region. The same procedure was applied at all particle flux points and the mean of the resulting histograms was plotted versus particle flux (in $\mathrm{MHz} / \mathrm{cm}^{2}$ ) as shown on Fig. 5). The uncertainty on the data points in the plot include statistical and systematic sources. The systematic uncertainty was determined by assuming no flux dependence below $80 \mathrm{kHz} / \mathrm{cm}^{2}$. Under this assumption, the standard deviation of the distribution of the average pulse height measurements for all flux points below this flux was used to quantify the systematic uncertainty.

\section{Conclusion and Outlook}

We have demonstrated the successful fabrication and operation of a 3D charged particle sensitive detector prototype based on poly-crystalline CVD diamond. The average charge collected by a continuous fiducial region of cells with successfully formed and connected electrodes is larger than the average charge collected by a planar detector and collected even with much smaller bias 


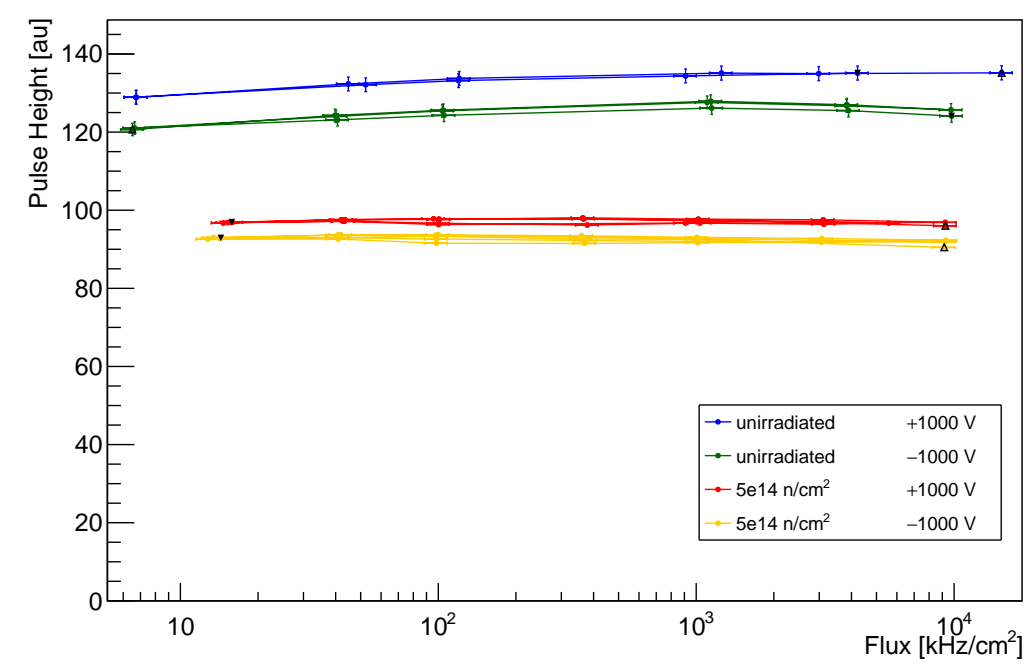

Figure 5: Average pulse height versus rate for an unirradiated and irradiated $\left(5 \times 10^{14} \mathrm{n}_{\mathrm{eq}} / \mathrm{cm}^{2}\right)$ pCVD diamond pad detector at positive and negative voltage. The pulse height units are arbitrary and the unirradiated and irradiated detector use different electronics, whose relative gains are being determined.

voltage. We have demonstrated that the average signal pulse height of pCVD diamond pad detectors irradiated up to the dose of $5 \times 10^{14} \mathrm{n}_{\mathrm{eq}} / \mathrm{cm}^{2}$ does not depend on the the particle flux up to $10 \mathrm{MHz} / \mathrm{cm}^{2}$. We plan to study the pulse height dependence of poly-crystalline and single-crystal CVD diamond sensors with pad and pixel electrodes further with higher irradiation doses up to $2 \times 10^{16} \mathrm{n}_{\mathrm{eq}} / \mathrm{cm}^{2}$.

\section{Acknowledgments}

The RD42 Collaboration would like to gratefully acknowledge the staff at CERN for allocating us the test beam time and for their help in setting up the excellent beam conditions. We would especially like to thank Henric Wilkins, the test beam coordinator, for his assistance in making our tests a success. We would also like to thank the beam line staff at the Paul Scherrer Institute (PSI) High Intensity Proton Accelerator. We would like to especially thank Konrad Deiters, Manuel Schwarz and Davide Reggiani of PSI for their expert and invaluable assistance in carrying out the diamond detector tests. We also extend our gratitude to Prof. Lin Li and David Whitehead of the Laser Processing Centre, University of Manchester for assisting in the production of the 3D diamond device. The research leading to these results received funding from the European Union's Horizon 2020 research and innovation programme under grant agreement No. 654168. This work was also partially supported by the Swiss National Science Foundation grant \#20FL20_ 154216, ETH grant 51 15-1, Royal Society Grant UF120106 and the U.S. Department of Energy through grant DE-SC0010061. 


\section{A. RD42 Author list}

A. Alexopoulos ${ }^{3}$, M. Artuso ${ }^{22}$, F. Bachmair ${ }^{26}$, L. Bäni ${ }^{26}$, M. Bartosik ${ }^{3}$, J. Beacham ${ }^{15}$, H. Beck ${ }^{25}$, V. Bellini' ${ }^{2}$, V. Belyaev ${ }^{14}$, B. Bentele ${ }^{21}$, E. Berdermann ${ }^{7}$, P. Bergonzo ${ }^{13}$, A. Bes ${ }^{30}$, J-M. Brom ${ }^{9}$, M. Bruzzi ${ }^{5}$, M. Cerv ${ }^{3}$, G. Chiodini ${ }^{29}$, D. Chren ${ }^{20}$, V. Cindro ${ }^{11}$, G. Claus ${ }^{9}$, J. Collot ${ }^{30}$, J. Cumalat ${ }^{21}$, A. Dabrowski ${ }^{3}$,

R. D'Alessandro ${ }^{5}$, W. de Boer ${ }^{12}$, B. Dehning ${ }^{3}$, C. Dorfer ${ }^{26}$, M. Dunser ${ }^{3}$, V. Eremin ${ }^{8}$, R. Eusebi ${ }^{27}$, G. Forcolin ${ }^{24}$, J. Forneris ${ }^{17}$, H. Frais-Kölbl ${ }^{4}$, K.K. Gan ${ }^{15}$, M. Gastal ${ }^{3}$, C. Giroletti ${ }^{19}$, M. Goffe ${ }^{9}$, J. Goldstein ${ }^{19}$, A. Golubev ${ }^{10}$, A. Gorišek ${ }^{11}$, E. Grigoriev ${ }^{10}$, J. Grosse-Knetter ${ }^{25}$, A. Grummer ${ }^{23}$, B. Gui ${ }^{15}$, M. Guthoff ${ }^{3}$, I. Haughton ${ }^{24}$, B. Hiti ${ }^{11}$, D. Hits ${ }^{26}$, M. Hoeferkamp ${ }^{23}$, T. Hofmann ${ }^{3}$, J. Hosslet ${ }^{9}$, J-Y. Hostachy ${ }^{30}$, F. Hügging ${ }^{1}$, C. Hutton ${ }^{19}$, H. Jansen ${ }^{3}$, J. Janssen ${ }^{1}$, H. Kagan ${ }^{15, \diamond}$, K. Kanxheri ${ }^{31}$, G. Kasieczka ${ }^{26}$, R. Kass ${ }^{15}$, F. Kassel ${ }^{12}$, M. Kis ${ }^{7}$, G. Kramberger ${ }^{11}$, S. Kuleshov ${ }^{10}$, A. Lacoste ${ }^{30}$, S. Lagomarsino ${ }^{5}$, A. Lo Giudice ${ }^{17}$, E. Lukosi ${ }^{28}$,

C. Maazouzi $^{9}$, I. Mandic ${ }^{11}$, C. Mathieu ${ }^{9}$, N. McFadden ${ }^{23}$, M. Menichelli ${ }^{31}$, M. Mikuž ${ }^{11}$, A. Morozzi ${ }^{31}$, J. Moss ${ }^{15}$, R. Mountain ${ }^{22}$, S. Murphy ${ }^{24}$, M. Muškinja ${ }^{11}$, A. $\mathrm{Oh}^{24}$, P. Oliviero $^{17}$, D. Passeri ${ }^{31}$, H. Pernegger ${ }^{3}$, R. Perrino ${ }^{29}$ F. Picollo ${ }^{17}$, M. Pomorski ${ }^{13}$, R. Potenza ${ }^{2}$, A. Quadt ${ }^{25}$, A. $\operatorname{Re}^{17}$, M. Reichmann ${ }^{26}$, G. Riley ${ }^{28}$, S. Roe $^{3}$, D. $\mathrm{Sanz}^{26}$, M. Scaringella ${ }^{5}$, D. Schaefer ${ }^{3}$, C.J. Schmidt ${ }^{7}$,

S. Schnetzer ${ }^{16}$, T. Schreiner ${ }^{4}$, S. Sciortino ${ }^{5}$, A. Scorzoni ${ }^{31}$,

S. Seidel ${ }^{23}$, L. Servoli ${ }^{31}$, B. Sopko ${ }^{20}$, V. Sopko ${ }^{20}$, S. Spagnolo ${ }^{29}$, S. Spanier ${ }^{28}$, K. Stenson ${ }^{21}$,
R. Stone ${ }^{16}$, C. Sutera ${ }^{2}$, A. Taylor ${ }^{23}$, M. Traeger ${ }^{7}$, D. Tromson ${ }^{13}$, W. Trischuk ${ }^{18, \diamond}$, C. Tuve ${ }^{2}$, L. Uplegger ${ }^{6}$, J. Velthuis ${ }^{19}$, N. Venturi ${ }^{18}$, E. Vittone ${ }^{17}$, S. Wagner ${ }^{21}$, R. Wallny ${ }^{26}$, J.C. Wang ${ }^{22}$, J. Weingarten ${ }^{25}$, C. Weiss ${ }^{3}$, T. Wengler ${ }^{3}$, N. Wermes ${ }^{1}$, M. Yamouni ${ }^{30}$, M. Zavrtanik ${ }^{11}$

${ }^{1}$ Universität Bonn, Bonn, Germany, ${ }^{2}$ INFN/University of Catania, Catania, Italy, ${ }^{3}$ CERN, Geneva, Switzerland, ${ }^{4}$ FWT, Wiener Neustadt, Austria, ${ }^{5}$ INFN/University of Florence, Florence, Italy, ${ }^{6}$ FNAL, Batavia, USA, ${ }^{7}$ GSI, Darmstadt, Germany, ${ }^{8}$ Ioffe Institute, St. Petersburg, Russia, ${ }^{9}$ IPHC, Strasbourg, France, ${ }^{10}$ ITEP, Moscow, Russia, ${ }^{11}$ Jožef Stefan Institute, Ljubljana, Slovenia,

12 Universität Karlsruhe, Karlsruhe, Germany,

${ }^{13}$ CEA-LIST Technologies Avancees, Saclay, France,

${ }^{14}$ MEPHI Institute, Moscow, Russia, ${ }^{15}$ The Ohio State University, Columbus, OH, USA, ${ }^{16}$ Rutgers University, Piscataway, NJ, USA, ${ }^{17}$ University of Torino, Torino, Italy,

18 University of Toronto, Toronto, ON, Canada,

${ }^{19}$ University of Bristol, Bristol, UK, ${ }^{20}$ Czech Technical Univ., Prague, Czech Republic, ${ }^{21}$ University of Colorado, Boulder, CO, USA, ${ }^{22}$ Syracuse University, Syracuse, NY, USA, ${ }^{23}$ University of New Mexico, Albuquerque, NM,

USA, ${ }^{24}$ University of Manchester, Manchester, UK,

${ }^{25}$ Universität Goettingen, Goettingen, Germany, ${ }^{26}$ ETH Zürich, Zürich, Switzerland, ${ }^{27}$ Texas A\&M, College Park Station, TX, USA, ${ }^{28}$ University of Tennessee, Knoxville, TN, USA, ${ }^{29}$ INFN-Lecce, Lecce, Italy, ${ }^{30}$ LPSC-Grenoble, Grenoble, France, ${ }^{31}$ INFN-Perugia, Perugia, Italy.

\section{References}

[1] G. Apollinari , I. Bejar Alonso, O. Brüning, M. Lamont, L. Rossi (editors), High-Luminosity Large Hadron Collider (HL-LHC) : Preliminary Design Report, CERN-2015-005 251 (2015).

[2] F. Bachmair, CVD Diamond Sensors In Detectors For High Energy Physics, Ph.D. Thesis ETH Zurich, (2016), http://dx.doi.org/10.3929/ethz-a-010748643

[3] F. Bachmair et al., A 3D Diamond Detector for Particle Tracking, Nucl. Instrum. Meth. A, 786, 97-104, (2015)

[4] S. Parker, C. Kenney, J. Segal, 3D - A proposed new architecture for solid-state radiation detectors, Nucl. Instr. and Meth. A, 395, 328, (1997)

[5] T. V. Kononenko, et al., Microsctructuring of diamond bulk by IR femtosecond laser pulses, Appl. Phys. A, 90, 645-651, (2008)

[6] O. Toker, S. Masciocchi, E. Nygård, A .Ritdge, P.Weilhammer, VIKING, a CMOS low noise monolithic 128 channel frontend for Si-strip detector readout, Nucl. Instrum. Meth. A, 340, 572-579, (1994)

[7] S. Schnetzer, et al. [RD42 Collaboration], Diamond Sensors for Energy Frontier Experiments, Proceedings of Science, Vertex 2013, 029, (2013) 
[8] E. Halkiadakis, et al., A Proposed Luminosity Monitor for CMS based on Small Angle Diamond Pixel Telescopes., Nucl. Instrum. Meth. A, 565, 284-289, (2006)

[9] PSI HIPA, High Energy Beam Lines,

http://www.psi.ch/abe/high-energy-beam-lines

[10] H. Chr. Kästli, M. Barbero, W. Erdmann, Ch. Hormann, R. Horisberger, et al., Design and performance of the CMS pixel detector readout chip, Nucl. Instrum. Meth. A, 565, 188-194, (2006).

[11] II-VI Incorporated, http://www.iiviinfrared.com/Optical-Materials/ cvd-diamond_substrates.html

[12] D. Przyborowski, J. Kaplon, and P. Rymaszewski, Design and Performance of the BCM1F Front End ASIC for the Beam Condition Monitoring System at the CMS Experiment, IEEE Trans. Nucl. Sci., 63 , 2300, (2016)

[13] S. Ritt, DRS4 Evaluation Board, http://www.psi . ch/drs/evaluation-board 